\title{
Adaptive EEG-Based Alertness Estimation System by Using ICA-Based Fuzzy Neural Networks
}

\author{
Chin-Teng Lin, Fellow, IEEE, Li-Wei Ko, I-Fang Chung, Teng-Yi Huang, Yu-Chieh Chen, Tzyy-Ping Jung, and \\ Sheng-Fu Liang
}

\begin{abstract}
Drivers' fatigue has been implicated as a causal factor in many accidents. The development of human cognitive state monitoring system for the drivers to prevent accidents behind the steering wheel has become a major focus in the field of safety driving. It requires a technique that can continuously monitor and estimate the alertness level of drivers. The difficulties in developing such a system are lack of significant index for detecting drowsiness and the interference of the complicated noise in a realistic and dynamic driving environment. An adaptive alertness estimation methodology based on electroencephalogram, power spectrum analysis, independent component analysis (ICA), and fuzzy neural network (FNNs) models is proposed in this paper for continuously monitoring driver's drowsiness level with concurrent changes in the alertness level. A novel adaptive feature selection mechanism is developed for automatically selecting effective frequency bands of ICA components for realizing an on-line alertness monitoring system based on the correlation analysis between the time-frequency power spectra of ICA components and the driving errors defined as the deviation between the center of the vehicle and the cruising lane in the virtual-reality driving environment. The mechanism also provides effective and efficient features that can be fed into ICA-mixture-model-based self-constructing FNN to indirectly estimate driver's drowsiness level expressed by approximately and predicting the driving error.
\end{abstract}

Index Terms-Alertness estimation, electroencephalogram (EEG), independent component analysis (ICA), ICA-mixture-model-based self-constructing fuzzy neural networks (ICAFNN), power spectrum analysis.

Manuscript received December 22, 2005; revised August 16, 2006. This work was supported in part by the Ministry of Education, Taiwan, under Grant EX-91-E-FAOE-4-4, Ministry of Economic Affairs, Taiwan, R.O.C. under Grant 93-17-A-02-S1-032, the National Science Council, Taiwan, R.O.C., under Grant NSC 94-2218-E-009-031- and MOE ATU Program 95W803E. This paper was recommended by Guest Editor P. C. Chung.

C.-T. Lin is with the Department of Electrical and Control Engineering, the Department of Computer Science, and the Brain Research Center, National Chiao-Tung University (NCTU), Hsinchu 300, Taiwan, R.O.C. (e-mail: ctlin@mail.nctu.edu.tw).

L.-W. Ko, T.-Y. Huang, and Y.-C. Chen are with the Department of Electrical and Control Engineering and the Brain Research Center, National Chiao-Tung University (NCTU), Hsinchu 300, Taiwan, R.O.C. (e-mail: 1wko@mail.nctu. edu.tw).

I-F. Chung is with the Institute of Bioinformatics, National Yang-Ming University, Taipei 112, Taiwan, R.O.C. (e-mail: ifchung@ym.edu.tw).

T.-P. Jung is with the Institute for Neural Computation, University of California, San Diego, CA 92037 USA, and also with the Department of Computer Science and the Brain Research Center, National Chiao-Tung University (NCTU), Hsinchu 300, Taiwan, R.O.C. (e-mail: jung@ sccn.ucsd.edu).

S.-F. Liang is with the Department of Computer Science and Information Engineering, National Cheng Kung University, Tainan 701, Taiwan, R.O.C., and also with the Brain Research Center, NCTU, Hsinchu 300, Taiwan, R.O.C. (e-mail: sfliang@mail2000.com.tw).

Digital Object Identifier 10.1109/TCSI.2006.884408

\section{INTRODUCTION}

D EVELOPMENT of the drowsiness monitoring technology for preventing accidents behind the steering wheel has become a major interest in the field of safety driving since drivers' fatigue is a causal factor in many accidents because of the marked decline in the drivers' abilities of perception, recognition, and vehicle control abilities while sleepy. Thus, developing accurate and noninvasive real-time driver drowsiness monitoring system would be highly desirable, particularly if this system can be further integrated into an automatic warning system. It is known that abundant information on physiological changes such as eye activity measures, heart rate variability (HRV), or particularly, the electroencephalogram (EEG) activities can relate with drowsiness [1]-[5]. Previous studies [6]-[8] showed that the eye blink duration and the blink rate typically increases while blink amplitude decreases as function of the cumulative time, and the saccade frequencies and velocities of electrooculogram (EOG) decline when people get drowsy. Although approaches based on EOG signals showed that eye-activity variations were highly correlated with the human fatigue and can accurately and quantitatively estimate alertness levels, the step size (temporal resolution) of those eye-activity based methods is relatively long (about $10 \mathrm{~s}$ ) to track slow changes in vigilance [9]. Contrarily, the step size of the EEG-based methods can be reduced to about $2 \mathrm{~s}$ to track second-to-second fluctuations in the subject's performance [10]-[17]. Since the computer power becomes faster and faster, it is practicable and appealing to know what information about human cognitive state and behavior are available through analyzing complex EEG signals. In [18], we constructed a virtual-reality (VR) based highway-driving environment to study drivers' cognitive changes during a long-term driving. A lane-keeping driving experiment was designed to indirectly quantify the driver's drowsiness level and a drowsiness estimation system combining the EEG power spectrum analysis, the principle component analysis (PCA) and the linear regression model was developed. In [19], the independent component analysis (ICA) [20]-[24] was used in the similar experiments to locate the optimal electrode placements for each individual. A total of ten frequency bands in two ICA components are selected and fed to the linear regression models to estimate driver's performance. In order to develop an on-line alertness estimation system and improve its performance, we develop a novel adaptive feature selection mechanism (AFSM) based on the correlation analysis between the subjects' driving errors 
and power spectra of the ICA components to automatically select effective features in this paper. In addition, the ICA-mixture-model-based fuzzy neural networks (ICAFNN) developed in [25] are employed as the drowsiness estimator to improve the prediction performance.

\section{EXPERIMENTAL SETUP}

\section{A. Virtual-Reality (VR)-Based Driving Environment}

A virtual-reality (VR) based highway-driving environment that was developed in our previous studies [18], [19] to investigate drivers' cognitive changes during a long-term driving was also used in this paper. It includes 3-D surround scenes projected by seven projectors and a real car mounted on a 6-degree-of-freedom Stewart platform. During the driving experiment, all scenes are moving according to the displacement of the car and the subject's wheel handling. The driving speed is fixed as $100 \mathrm{~km} / \mathrm{hr}$ and the car is randomly and automatically drifted away from the center of the cruising lane to mimic the consequences of a non-ideal road surface. We asked the subject to keep the car on the third cruising lane (from left to right counted). While the subject is alert, his/her response time will be short and deviation of the car will be small; otherwise the subject's response time and the car deviation will be slow and long. In this driving experiment, the VR-based freeway scene provides only one car driven on the road without any other event stimuli to simulate a monotonous and unexciting task that will make drivers fallen asleep.

\section{B. Subjects}

A total of ten subjects (ages from 20 to 40 years, $29.8 \pm 5.9$ years old) participated in the VR-based highway driving experiments. Each subject completed a training session in one day and testing sessions on the other days. To maximize the opportunities to get valuable data for our study, all the experiments were conducted in the early afternoons after lunch. Statistical reports [26] showed that people often get drowsy within one hour of continuous driving during these periods, indicating that drowsiness is not necessarily caused by long driving-hours. On the first day, participants were told of the general features of the driving task, completed necessary informed consent material, and then started with a 15 to 45 -min practice to keep the car at the center of the cruising lane by maneuvering the car with the steering wheel. Subjects reported this amount of practice to be sufficient to train participants to asymptote on the task. After practicing, participants were wired with EEG electrodes and began a 45-min lane-keeping driving task. Participants returned on a different day to complete the other 45-min driving session for cross-session test.

\section{Data Acquisition}

The physiological data acquisition uses 33 sintered $\mathrm{Ag} / \mathrm{AgCl}$ $\mathrm{EEG} / \mathrm{EOG}$ electrodes with a unipolar reference at right earlobe and 2 ECG channels in bipolar connection placed on the chest. All the EEG/EOG electrodes were placed based on a modified International 10-20 system and refer to right ear lobe. Before data acquisition, the contact impedance between EEG electrodes and cortex was calibrated to be less than $5 \mathrm{k} \Omega$. We use the

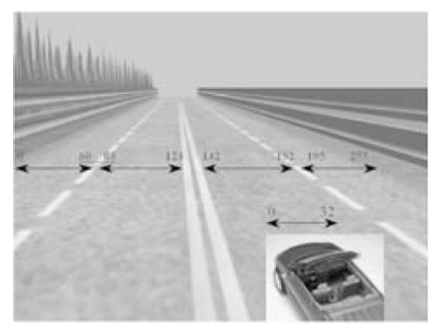

(a)

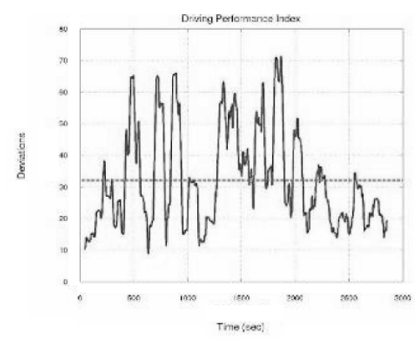

(b)
Fig. 1. (a) VR-based freeway scene. (b) Driving error. Horizontal dashed line means the "dangerous" boundary.

Scan NuAmps Express system (Compumedics Ltd., VIC, Australia) to simultaneously record the EEG/EOG/ECG data and the deviation between the center of the vehicle and the center of the cruising lane triggered by the VR program. The EEG data were recorded with 16-bit quantization level at sampling rate $500 \mathrm{~Hz}$ and the recording are down-sampled to sampling rate $250 \mathrm{~Hz}$ for the simplicity of data processing. Then, EEG data were preprocessed using a simple low-pass filter with a cutoff frequency of $50 \mathrm{~Hz}$ to remove the line noise $(60 \mathrm{~Hz}$ and its harmonic) and other high-frequency noise for further analysis.

\section{Alertness Measurement}

In order to investigate the relationship between the measured EEG signals and subject's cognitive state, and to quantify the level of the subject's alertness, we defined the subject's alertness level index $D_{p}(t)$ as the deviation between the center of the vehicle and the center of the cruising lane as an indirect measurement of the subject's alertness level. Our pilot studies showed that when the subject is drowsy (checked from video recordings and subjects' reports), the derivation error of car drift increases, and vice versa. Since the fluctuates of drowsiness level with cycle lengths were longer than 4 min [12]-[15], the alertness level index were smoothed using a causal 90-s square moving-average filter advancing at 2-s steps to eliminate variance at cycle lengths shorter than $1-2 \mathrm{~min}$ as (1). It is noted that the step size of eye-activity based approaches is about $10 \mathrm{~s}$ [9]. It means that the temporal resolution of our method is 5 times higher than that of the eye-activity based approaches

$$
D_{p}(t)=\frac{1}{M_{e}} \sum_{i=1+2(t-1) \Omega_{s}}^{M_{e}+2(t-1) \Omega_{s}}\left|e\left(M_{e}+2(t-1) \Omega_{s}-i\right)\right|
$$

where $M_{e}$ is the 90 -s window length $\left(M_{e}=90 \times \Omega_{s}\right), \Omega_{s}$ is the sampling rate, and $e(t)$ denotes the distance between the center of the vehicle and the center of the cruising lane at time $t$. Fig. 1 shows the designed VR-based freeway scene and the smoothed alertness level index. The red numbers in Fig. 1(a) depict the widths in pixels of the four lanes from left to right in VR frontal scene. In our case, each lane covers 60 pixels and the width of the car is 32 pixels. We can also convert the distance of each pixel into the width of the real road. Fig. 1(b) plots the time course of deviation (alertness level) across time (in seconds). Each experiment lasted about $45-\min$ (2700 s). The vertical axis represents the deviation of the car in pixel. When the deviation 


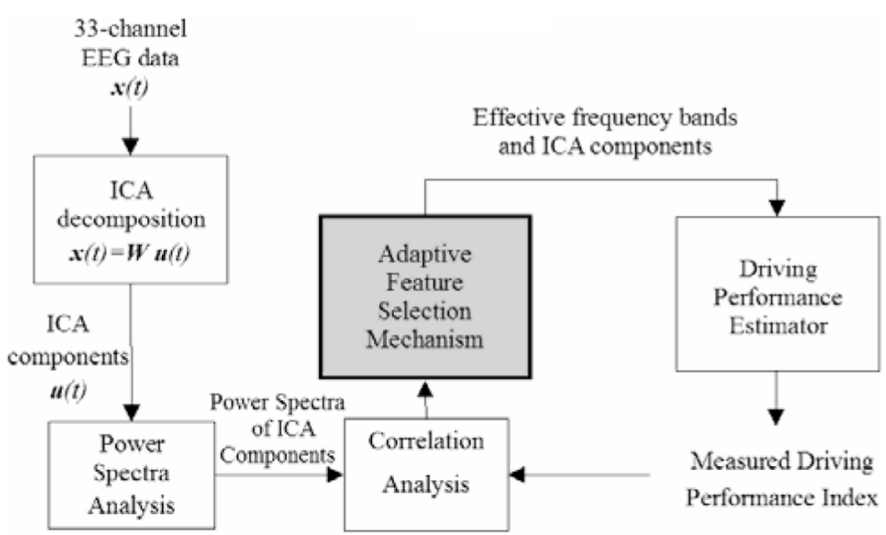

Fig. 2. Flowchart of data processing procedures of the proposed drowsiness.

is greater than 32 pixels [horizontal dashed line in Fig. 1(b)], the car was completely off the cruising lane which could easily lead to accidents. Participants who demonstrated waves of drowsiness containing two or more micro-sleeps in both sessions were selected as successful subjects. Based on these criteria, five participants (ten sessions) were selected for further modeling and cross-session testing.

\section{DATA ANALYSIS}

The proposed data analysis procedure for drowsiness estimation is shown in Fig. 2. Analytical modules are detailed below.

\section{A. Independent Component Analysis}

ICA is a signal processing technique that finds a linear mapping matrix or unmixing matrix $W$ such that the unknown unmixed signals of the dimension $N, U(t)=\left[u_{1}(t), \ldots, u_{N}(t)\right]^{T}$ could be separated from their mixtures, $X(t)$, that is $Y(t)=$ $W^{-1} X(t)$. The ICA methods were extensively applied to blind source separation problem since 1990s [17], [20]-[24]. Subsequent technical reports [27]-[33] demonstrated that ICA was a suitable solution to the problem of EEG source segregation, identification, and localization.

In this study, we used an extended version of informax algorithm of Bell and Sejnowski [19], [22] that can separate sources with either super- or sub-Gaussian distributions, to decompose distinct brain activities. It has also been used in our previous study [19]. Fig. 3 shows the scalp topographies of ICA back-projection matrix $W^{-1}$ of subject 2 and the log bandpower spectra of all ICA components. As shown in Fig. 3, most of the eyemovement artifacts are isolated to components $1-3$, while ICA components 8, 17, 27 and etc. are drowsiness related (based on the correlation analysis below).

\section{B. Power Spectrum Analysis}

Analysis of changes in spectral power and phase can characterize the perturbations in the oscillatory dynamics of ongoing EEG. Moving-averaged spectral analysis of the drowsiness related component data was then accomplished using a 750-point Hanning window with 250-point overlap, i.e., stepping in 2 s. Each 750-point epoch was further divided into several 125-point frames using Hanning windows with 25-point step size again. Windowed 125-point frames were extended to 256 points by zero-padding to calculate its power spectrum by using a 256-point fast Fourier transform (FFT), resulting in power-spectrum density estimation with a frequency resolution near $1 \mathrm{~Hz}$. Then, we averaged the power spectrum of all the sub-epochs within each epoch. Previous studies [18], [19] showed that the EEG spectral amplitudes correlated with the wake-sleep transition more linearly in the logarithmic scale than in the linear scale. Therefore, the averaged power spectrum of each epoch was normalized to logarithmic scale to linearize these multiplicative effects. Since the fluctuates of drowsiness level with cycle lengths were longer than 4 min [13], [14], the alertness level was smoothed using a causal 90-s square moving-average filter advancing at 2-s steps to eliminate variance at cycle lengths shorter than 1-2 min.

\section{Correlation Analysis}

In order to find the relationship between the brain activities and the subject's alertness level and to extract the effective frequency bands of drowsiness related components, we computed the correlation coefficient between two time courses of the alertness level index and the concurrent power changes at each frequency of the ICA components by using the Pearson correlation coefficient to form a correlation spectrum as follows:

$$
\operatorname{Corr}_{33 \times 60}=\left[\begin{array}{cccc}
c_{1,1} & c_{1,2} & \cdots & c_{1,60} \\
c_{2,1} & c_{2,2} & \cdots & c_{2,60} \\
\vdots & \vdots & \ddots & \vdots \\
c_{33,1} & c_{33,2} & \cdots & c_{33,60}
\end{array}\right]
$$

and

$$
\begin{gathered}
c_{i, k}=\frac{\sum_{n}\left(X_{i}(n, k)-\bar{X}_{i}(k)\right)\left(D_{p}(n)-\bar{D}_{p}\right)}{\sqrt{\sum_{n}\left(X_{i}(n, k)-\bar{X}_{i}(k)\right)^{2}} \sqrt{\sum_{n}\left(D_{p}(n)-\bar{D}_{p}\right)^{2}}}, \\
i=1, \ldots, 33 \\
k=1, \ldots, 60
\end{gathered}
$$

where $D_{p}(n)$ is the time series of the alertness level index, $X_{i}(n, k)$ is the time-frequency series of the $i$ th ICA component, $n$ is the time stepping size in $2 \mathrm{~s}$, and $k$ is the frequency index $(k=1,2, \ldots, 60) \cdot \bar{X}_{i}(k)$ and $\bar{D}_{p}$ are the expected value of $X_{i}(n, k)$ and $D_{p}(n)$.

\section{Adaptive Feature Selection Mechanism}

In order to automatically select the drowsiness related features, an adaptive feature selection mechanism based on the correlation coefficients between log bandpower of the drowsiness related components and the subject's alertness level index (SALI) is proposed.

We use the correlation spectra of subject 2 as an example to illustrate the proposed adaptive feature selection mechanism (Fig. 4). First, we compute the correlation coefficient between two time series in alertness level index and the concurrent changes in the ICA power spectrum at each frequency band (1-60 Hz in 33 ICA components) by (2). Then, we sort the correlation coefficients in each row vectors of matrix Corr 


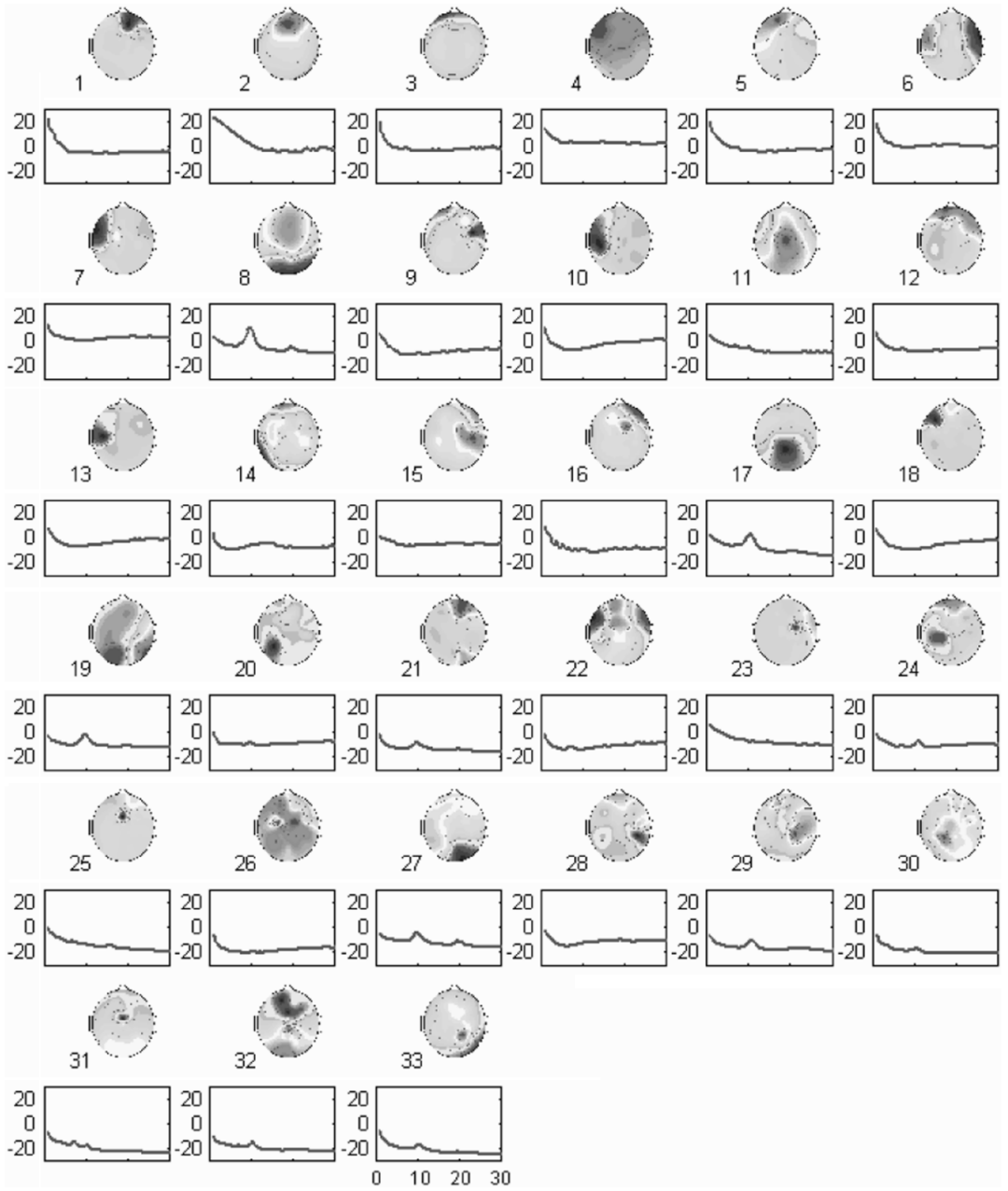

Fig. 3. Scalp topographies and the corresponding log bandpower spectra of all ICA components.

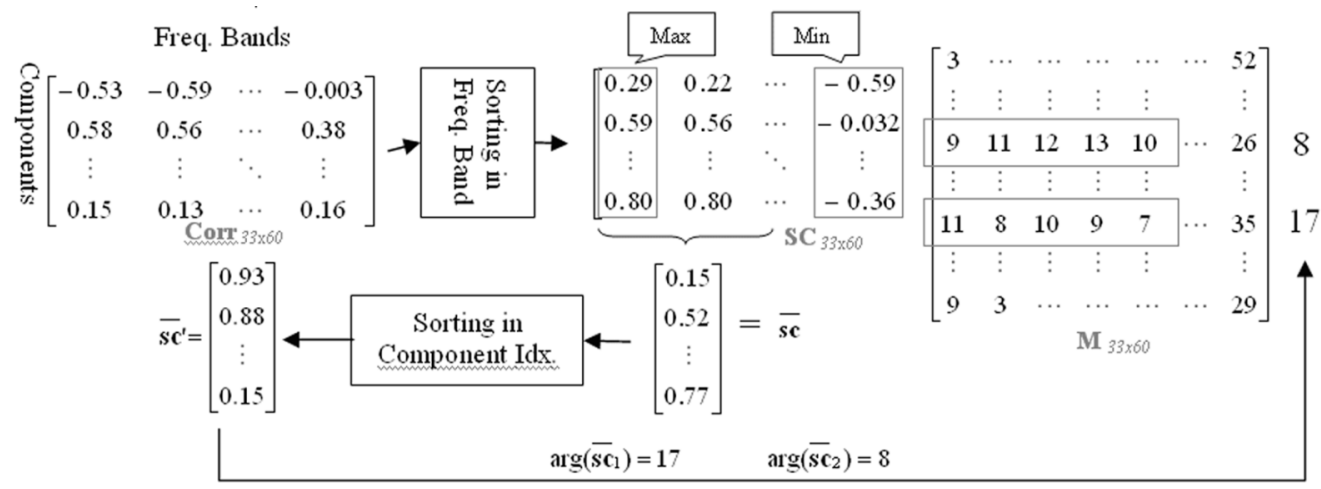

Fig. 4. Using subject 2 as an example to illustrate the adaptive feature selection mechanism. Note that the band power of ICA component 8 between 9 and $13 \mathrm{~Hz}$ and component 17 between 7 and $11 \mathrm{~Hz}$ are selected as input feature of the estimators.

by descending order so that each element in the first column of $\mathbf{S C}$ is the maximum correlation coefficient of the row it belongs to. $\mathbf{M}$ is the corresponding index matrix of $\mathbf{S C}$. After summing the first five elements in each row of $\mathbf{S C}$, we get the $\overline{\mathbf{s c}}$ vector. According to $\overline{\mathbf{s c}}^{\prime}$, which is the sorted version of $\overline{\mathbf{s c}}$, we can find that the averaged correlation coefficients of two most drowsiness-related ICA components of subject 2 are 0.93 and 0.88 . Referring to the index matrix $\mathbf{M}$, these two components are ICA components 8 and 17 and their selected frequency bands are 9-13 and 7-11 Hz, respectively.

\section{E. ICAFNNS}

An ICAFNN [25] shown in Fig. 5 was developed and performed as the alertness level estimator in the study. The ICAFNN is a novel FNN and it can construct itself with an economic network size, and the learning speed as well as the 


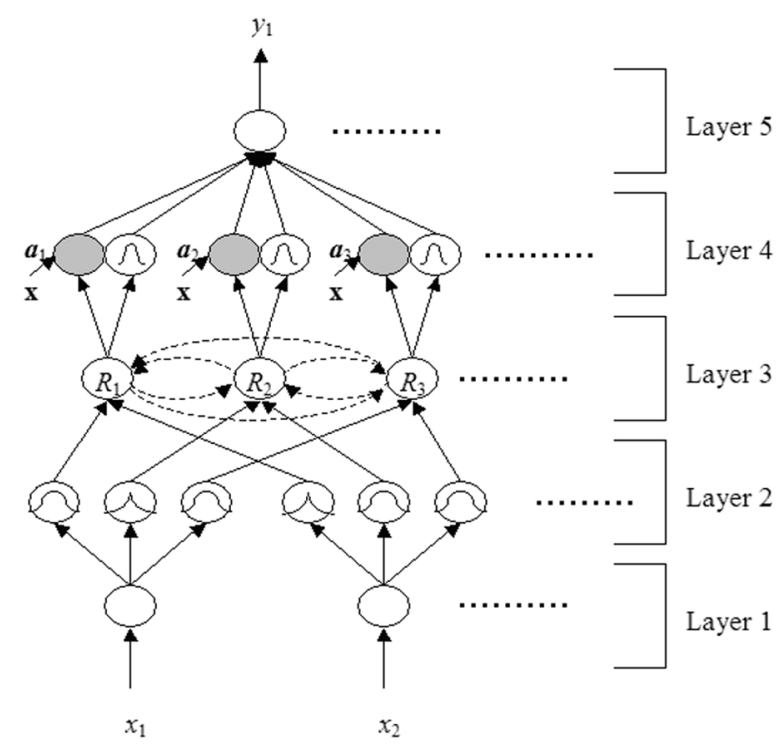

Fig. 5. Five layers of ICAFNN performing the functions of input clustering, fuzzy-rule reasoning, and parameter/structure learning.

modeling ability [25]. This five-layered network realizes a fuzzy inference system (FIS) of the following form:

$$
\begin{array}{ll}
\text { Rule } i: & \text { IF } x_{1} \text { is } A_{1}^{i} \text { and } \ldots x_{j} \text { is } A_{j}^{i} \ldots \text { and } x_{n} \text { is } A_{n}^{i}, \\
& \text { THEN } y_{i} \text { is } m_{0 i}+a_{1} x_{1}+a_{j i} x_{j}+\ldots
\end{array}
$$

where the current input data vector is $\mathbf{x}=\left[x_{1}, \ldots, x_{n}\right]^{T}, n$ is the number of input dimension, $A_{j}^{i}$ is a fuzzy set, $m_{0 i}$ is the center of a symmetric membership function on $y_{i}$, and $a_{j i}$ is a consequent parameter. The functions of each layer are illustrated as follows.

Layer 1: It only transmits input values to the next layer directly.

Layer 2: In this layer, the output $\mathbf{x}$ from Layer 1 is projected into the independent axes obtained by the on-line ICA mixture model such that

$$
\mathbf{s}_{j}=\mathbf{B}_{j} \mathbf{x}_{j}
$$

where $\mathbf{x}_{j}=\mathbf{x}-\mathbf{m}_{j}, \mathbf{B}_{j}$, and $\mathbf{m}_{j}$ are the basis matrix and mean vector, respectively, determined by the on-line ICA mixture model [25], $j=1,2, \ldots, J(t)$ and $J(t)$ is the number of clusters at time $t$.

With the choice of non-Gaussian membership function, the operation performed in this layer is

$$
\mathbf{u}_{i}^{(2)}=\mathbf{s}_{i} \quad \text { and } \quad f\left[u_{i j}^{(2)}\right]=p\left(u_{i j}^{(2)}\right)
$$

where $\mathbf{u}_{j}^{(2)}$ is inputs to node $i$ in layer $2, f$ is an integration function provides the node input for this node, the $p\left(u_{i j}\right)$ is proportional to super-Gaussian or sub-Gaussian, and the node output is a function of its node input

$$
o_{i}^{(2)}=a^{(2)}(f)=f
$$

where $a(\cdot)$ denotes the activation function. These notations will be used in the following equations. With the transformation of input coordinates, rule format in (4) should be modified as

$$
\begin{aligned}
& \text { Rule } i \text { IF } s_{i 1}=\sum_{k=1}^{n} b_{1 k}^{i}\left(x_{k}-m_{i k}\right)+m_{i k} \text { is } A_{i 1} \\
& \text { and } \ldots s_{i j}=\sum_{k=1}^{n} b_{j k}^{i}\left(x_{k}-m_{i k}\right)+m_{i k} \text { is } A_{i j}, \ldots \\
& \text { and } s_{\text {in }}=\sum_{k=1}^{n} b_{n k}^{i}\left(x_{k}-m_{i k}\right)+m_{i k} \text { is } A_{\text {in }} \\
& \text { THEN } y_{i} \text { is } m_{0 i}+a_{1 i} x_{1}+\cdots+a_{j i} x_{j}+\cdots
\end{aligned}
$$

where $b_{n k}^{i}$ the $(n, k)$ th element of $\mathbf{B}_{i}, \mathbf{B}_{i} \in \Re^{n \times n}$ is the transformation matrix for rule $i$, and $\mathbf{s}_{i} \in \Re^{n}$ are the newly generated input variables and it is called the sources in ICA.

The linguistic implication $\mathbf{A}_{i j}$ is now implicated by the new variable $s_{i j}$, which is a linear combination of the original variables. It is observed that the membership functions cover distribution of transformed data well such that a single fuzzy rule can associate the transformed region with its output region (consequent) properly.

Layer 3: A node in this layer represents one fuzzy rule and performs precondition matching of a rule. Here, we use the following AND operation for each Layer-2 node

$$
f\left[u_{i}^{(3)}\right]=\prod_{i} u_{i}^{(3)} \quad \text { and } \quad o_{i}^{(3)}=a^{(3)}(f)=\frac{f}{\sum_{j=1}^{J(t)} f_{j}} .
$$

The output of a Layer-3 node represents the firing strength of the corresponding fuzzy rule.

Layer 4: This layer is called the consequent layer. Two types of nodes are used in this layer as shown in Fig. 5. The node denoted by a blank circle is the essential node representing a fuzzy set of the output variable. As to the shaded node, each node in Layer 3 has its own corresponding shaded node in Layer 4. One of the inputs to a shaded node is the output delivered from Layer 3 and the other inputs are the input variables from Layer 1. Combining these two types of nodes in Layer 4, we obtain the whole function performed by this layer as

$$
\begin{aligned}
f\left[u_{i}^{(4)}\right] & =u_{i}^{(4)} \text { and } o_{i}^{(4)}=a^{(4)}(f) \\
& =\left(a_{0 i}+\sum_{j} a_{j i} x_{j}\right) f
\end{aligned}
$$

where $a_{0 i}=m_{0 i}$ is the center of output membership function and $a_{j i}$ is the corresponding parameter.

Layer 5: Each node in this layer corresponds to one output variable and acts as a defuzzifier with

$$
f\left[u_{i}^{(5)}\right]=\sum_{i} u_{i}^{(5)} \quad \text { and } \quad y_{i}=a^{(5)}(f)=f .
$$

Two types of learning-structure and parameter learning are used concurrently for constructing the ICAFNN [25]. The on-line ICA mixture model is used to realize the precondition and consequent structure identification of the proposed FNN. For the parameter learning, the parameters of the linear equations in the consequent parts are adjusted by the backpropagation rule to minimize a given cost function and the parameters in the precondition part are adjusted by the on-line 


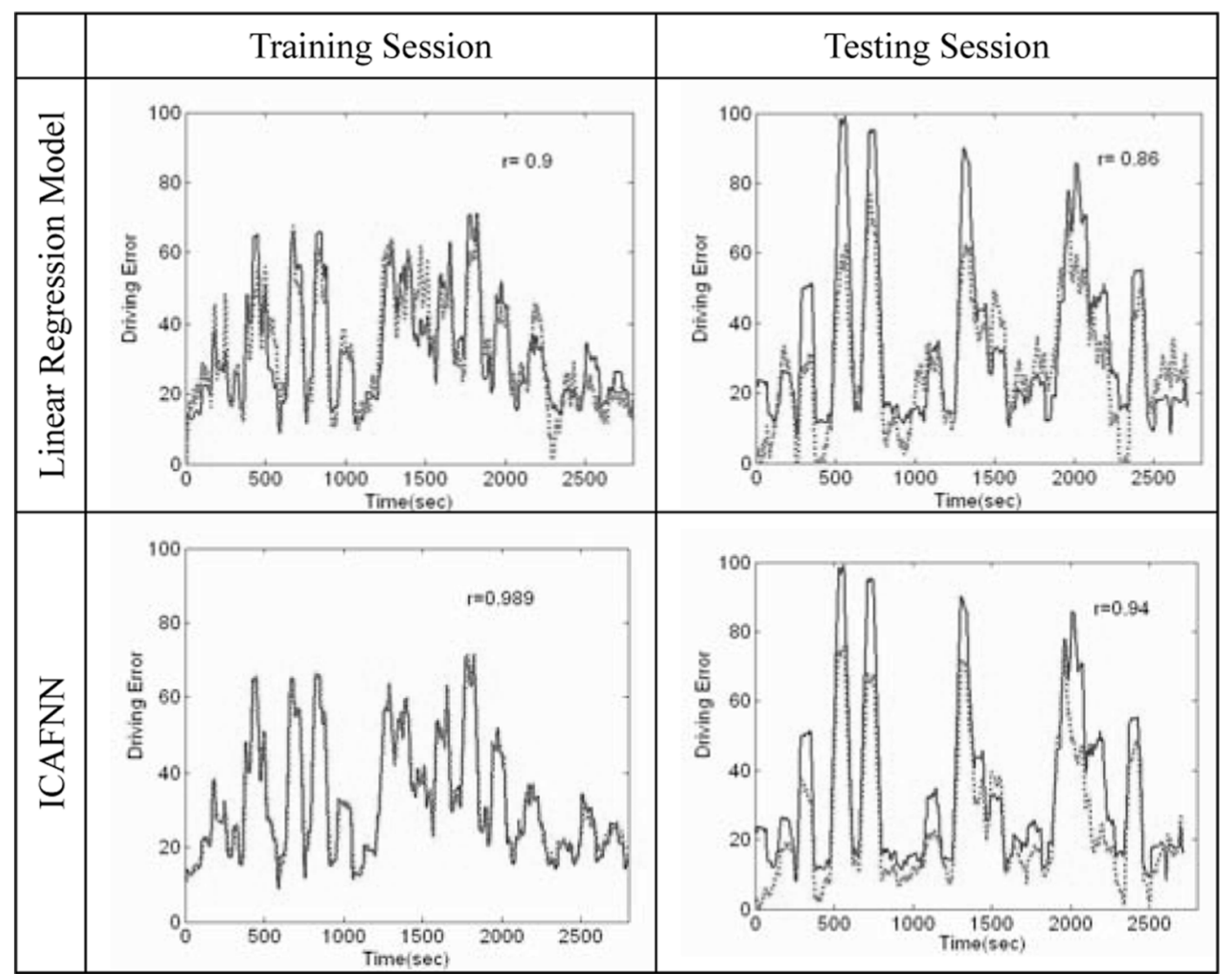

Fig. 6. Alertness level estimates for training/testing sessions of subject 2, based on a linear regression model (dot line, top panels) with subband log power of ICA components at 8-12 Hz, over plotted against actual driving performance time series for the session (solid line). The correlation coefficient between the two time series is $r=0.91$ in the training session and $r=0.89$ in the testing session. Bottom panels show the results in the same subject based on ICAFNN, the correlation coefficient between the two time series (dot and solid lines) increase to 0.989 in the training session, and 0.94 in the testing session, respectively.

ICA mixture model. There are no rulesin this network initially. They are created dynamically as learning proceeds upon receiving on-line incoming training data by performing the following learning processes simultaneously: 1) input/output space partitioning; 2) construction of fuzzy rules; 3 ) optimal consequent structure identification; 4) parameter identification. In the above, learning processes steps 1)-3) belong to the structure learning phase and step 4) belongs to the parameter learning phase [25].

\section{RESUlts AND Discussion}

\section{A. Adaptive Feature Selection}

According to Fig. 2, after ICA training and spectral analysis for each ICA components, we computed the spectral correlations between changes in the ICA log subband power spectrum and alertness level index. We then applied the proposed AFSM (Section III-D) to automatically select the features for drowsiness estimation. The selected features for five different subjects are shown in Table I. The features selected by the method in [19] are also included in Table I for comparison. As can be seen, two methods might select different components.

In general, the drowsiness-related regions are mainly in the parietal and occipital lobes. In [19], the optimal frequency bands were selected according to the correlation coefficients between ICA power spectra and drowsiness index and iteratively testing
TABLE I

COMPARISONS OF FEATURES SELECTED BY THE METHOD IN [19] AND THE AFSM CORRESPONDING TO DIFFERENT SUBJECTS

\begin{tabular}{|c|c|c|c|c|c|}
\hline & $\overline{\text { SUBJECT1 }}$ & $\overline{\text { SUBJECT2 }}$ & 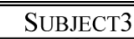 & SUBJECT4 & $\overline{\text { SUBJECT5 }}$ \\
\hline 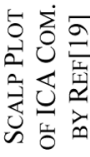 & & & & & $\therefore h$ \\
\hline $\begin{array}{l}\text { Freq. } \\
\text { Bands }\end{array}$ & $5 \sim 9 \mathrm{~Hz}$ & $8 \sim 12 \mathrm{~Hz}$ & $10 \sim 14 \mathrm{~Hz}$ & $4 \sim 8 \mathrm{~Hz}$ & $8 \sim 12 \mathrm{~Hz}$ \\
\hline 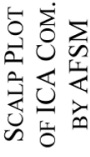 & & & & & \\
\hline $\begin{array}{l}\text { Freq. } \\
\text { Bands }\end{array}$ & $4 \sim 8 \mathrm{~Hz}$ & $5 \sim 9 \mathrm{~Hz}$ & $9 \sim 13 \mathrm{~Hz}$ & $7 \sim 11 \mathrm{~Hz}$ & $\begin{array}{c}10 \sim 14 \\
\mathrm{~Hz}\end{array}$ \\
\hline
\end{tabular}

by the linear regression model (LRM). On the contrary, the proposed AFSM is developed for one-path selection of effective frequency bands and the ICA components for realizing an on-line alertness monitoring system. In order to compare the performance of these two feature selection methods, the features are used as inputs of the linear regression models for driver's 
TABLE II

COMPARISONS OF DIFFERENT ALERTNESS LEVEL ESTIMATION APPROACHES INCLUDING LINEAR REGRESSION MODELS (LRM) USING THE FEATURES SELECTED BY THE METHOD IN [19], BY AFSM, AND THE ICAFNN MODEL USING THE FEATURES SELECTED BY AFSM FOR FIVE DIFFERENT SUBJECTS

\begin{tabular}{cccccccc}
\hline \hline \multicolumn{2}{c}{ SUBJECT } & 1 & 2 & 3 & 4 & 5 & $\begin{array}{c}\text { AVERAGE } \\
\%\end{array}$ \\
\hline $\begin{array}{c}\text { MANUAL } \\
\text { +LRM }\end{array}$ & TRAINING & $77 \%$ & $91 \%$ & $93 \%$ & $80 \%$ & $90 \%$ & $86.2 \pm 7.2$ \\
IN [19] & TESTING & $91 \%$ & $89 \%$ & $92 \%$ & $89 \%$ & $80 \%$ & $88.2 \pm 4.8$ \\
\hline AFSM+ & TRAINING & $92 \%$ & $91 \%$ & $93 \%$ & $84 \%$ & $90 \%$ & $90.0 \pm 3.6$ \\
LRM & TESTING & $91 \%$ & $88 \%$ & $92 \%$ & $82 \%$ & $80 \%$ & $86.6 \pm 5.4$ \\
\hline AFSM + & TRAINING & $97 \%$ & $98.9 \%$ & $97 \%$ & $99 \%$ & $99 \%$ & $98.2 \pm 1.0$ \\
ICAFNN & TESTING & $93 \%$ & $94 \%$ & $91 \%$ & $92 \%$ & $87 \%$ & $91.3 \pm 2.7$
\end{tabular}

alertness level estimation, as shown in Table II. The mean correlation between actual alertness level time series and within-session estimation by using the features selected by AFSM is $90 \%$, whereas the mean correlation coefficient between actual alertness level and cross-session estimation is $86.6 \%$. The average performance of the AFSM is closed to the performance using the optimal features. It can also be found that some testing results are better than the performance of the training sessions due to the repeatedly testing procedure. Fig. 6 shows the estimated alertness level of training/testing sessions of subject 2 obtained by the linear regression model with the feature selected by AFSM technique. These results demonstrate that the feature selected by the one-path selection method AFSM can also reach excellent performance in estimating driving error without the repeatedly testing process.

The performance of the proposed ICA-based AFSM is also compared favorably to the principle component analysis (PCA) based approach developed in [18] that used 50 PCA components as the input features of the linear regression model for drowsiness estimation. The mean correlation coefficient between actual alertness level and cross-session estimation of the PCAbased method is $0.53 \pm 0.12$ for the same 10 sessions.

\section{B. EEG-Based ICA-Mixture-Model-Based Fuzzy Neural Networks (ICAFNN) for Driver Alertness Estimation}

In this study, we fed the features selected by AFSM into the ICAFNN for subject's alertness level estimation. The ICA weight matrices obtained from the training sessions were used to spatially filter the features in the testing sessions so that training/ testing data were processed in the same way before feeding to the estimation models for the same subject. Fig. 6 shows that the estimated and actual alertness level index of training/testing sessions of subject 2 matched well with the actual alertness level ( $r=98.9 \%$ in the training session and $94 \%$ in the testing session, respectively). Table II (bottom row) summarizes the performance of alertness level estimation obtained by the ICAFNN model across ten sessions of five subjects. The mean correlation between actual and estimated alertness level time series is $98.2 \pm 1.0 \%$, whereas the mean correlation coefficient in cross-session testing is $91.3 \pm 2.7 \%$.

\section{CONCLUSION}

In this study, an adaptive EEG-based drowsiness estimation technology that combines ICA, power-spectrum analysis, AFSM, and ICAFNN is proposed to continuously, indirectly estimate/predict fluctuations in human alertness level indexed by alertness level measurement, expressed as deviation between the center of the vehicle and the center of the cruising lane in a virtual-reality based driving environment. The AFSM can automatically select effective features based on the correlation analysis between the power spectra of drowsiness related components and the driving errors. The proposed ICAFNN can accurately estimate driver's individual alertness level using ten sub-band power spectra of two ICA components selected by AFSM. The computational methods developed in this study can lead to on-line monitoring of human operators' cognitive state in attention-critical settings.

\section{ACKNOWLEDGMENT}

The authors would like to thank Mrs. J.-R. Duann, H.-Y. Hsieh, J.-L. Jeng, and Ms. M. Lin for their great help with developing and operating the experiments.

\section{REFERENCES}

[1] R. S. Huang, C. J. Kuo, L. L. Tsai, and O. T. C. Chen, "EEG pattern recognition arousal states detection and classification," in Proc. IEEE Conf. Neural Netw., Jun. 1996, vol. 2, pp. 641-646.

[2] A. Vuckovic, V. Radivojevic, A. C. N. Chen, and D. Popovic, "Automatic recognition of alertness and drowsiness from EEG by an artificial neural network," Med. Eng. Phys., vol. 24, pp. 349-360, 2002.

[3] S. Roberts, I. Rezek, R. Everson, H. Stone, S. Wilson, and C. Alford, "Automated assessment of vigilance using committees of radial basis function analysers," Proc. IEEE Sci. Meas. Technol., vol. 147, pp. 333-338, Nov. 2000.

[4] K. B. Khalifa, M. H. Bedoui, R. Raytchev, and M. Dogui, "A portable device for alertness detection," in Proc. 2000 1st Annual Int. IEEEEMBS Special Topic Confe. Microtechnolog. Med. Biol., Oct. 2000, pp. 584-586.

[5] B. J. Wilson and T. D. Bracewell, "Alertness monitor using neural networks for EEG analysis," in Proc. 2000 IEEE Signal Process. Soc. Workshop on Neural Netw. Signal Process., Dec. 2000, vol. 2, pp. 814-820.

[6] J. A. Stern, D. Boyer, and D. Schroeder, "Blink rate: Possible measure of fatigue," Human Factors, vol. 36, pp. 285-297, 1994.

[7] D. Schmidt, L. A. Abel, L. F. Dell'Osso, and R. B. Daroff, "Saccade velocity characteristics: Intrinsic variability and fatigue," Aviation Space Environ. Med., vol. 50, pp. 393-395, 1979.

[8] D. K. McGregor and J. A. Stern, "Time on task and blink effects on saccade duration," Ergonomics, vol. 39, pp. 649-660, 1996.

[9] K. Van Orden, T. P. Jung, and S. Makeig, "Combined eye activity measures accurately estimate changes in sustained visual task performance," Biol. Psychol., vol. 52, no. 3, pp. 221-40, 2000.

[10] K. Van Orden, W. Limbert, S. Makeig, and T. P. Jung, "Eye activity correlates of workload during a visualspatial memory task," Human Factors, vol. 43, no. 1, pp. 111-121, 2001.

[11] M. Matousek and I. Petersen, "A method for assessing alertness fluctuations from EEG spectra,” Electroencephalogr. Clin. Neurophysiol., vol. 55, no. 1, pp. 108-113, 1983.

[12] S. Makeig and T. P. Jung, "Changes in alertness are a principal component of variance in the EEG spectrum," NeuroReport, vol. 7, p. 21316, 1995.

[13] T. P. Jung, S. Makeig, M. Stensmo, and T. J. Sejnowski, "Estimating alertness from the EEG power spectrum," IEEE Trans. Biomed. Eng., vol. 44, no. 1, pp. 60-69, Jan. 1997.

[14] S. Makeig and M. Inlow, "Lapses in alertness: Coherence of fluctuations in performance and EEG spectrum," Electroencephalogy. Clin. Neurophysiol., vol. 86, pp. 23-35, 1993.

[15] S. Makeig and T. P. Jung, "Tonic, phasic and transient EEG correlates of auditory awareness in drowsiness," Cogn. Brain Res., vol. v, no. 4, pp. $15-25,1996$.

[16] M. Treisman, "Temporal rhythms and cerebral rhythms," in Timing and Time Perception, J. Gibbon and L. Allan, Eds. New York: Academic, 1984, vol. 423, pp. 542-565.

[17] J. Beatty, A. Greenberg, W. P. Deibler, and J. O'Hanlon, “Operant control of occipital theta rhythm affects performance, in a radar monitoring task," Science, vol. 183, pp. 871-873, 1974.

[18] C. T. Lin, R. C. Wu, T. P. Jung, S. F. Liang, and T. Y. Huang, "Estimating alertness level based on EEG spectrum analysis," EURASIP J. Appl. Signal Process., vol. 2005, no. 19, pp. 3165-3174, Mar. 2005. 
[19] C. T. Lin, R. C. Wu, S. F. Liang, W. H. Chao, Y. J. Chen, and T. P. Jung, "EEG-based drowsiness estimation for safety driving using independent component analysis," IEEE Trans. Circuits Syst. I, Reg. Papers, vol. 52, no. 12, pp. 2726-2738, Dec. 2005.

[20] C. Jutten and J. Herault, "Blind separation of sources I. An adaptive algorithm based on neuromimetic architecture," Signal Process., vol. 24, pp. 1-10, 1991.

[21] P. Comon, "Independent component analysis-A new concept?," Signal Process., vol. 36, pp. 287-314, 1994.

[22] A. J. Bell and T. J. Sejnowski, "An information-maximization approach to blind separation and blind deconvolution," Neural Comput., vol. 7, pp. 1129-1159, 1995.

[23] M. Girolami, "An alternative perspective on adaptive independent component analysis," Neural Comput., vol. 10, pp. 2103-2114, 1998.

[24] T. W. Lee, M. Girolami, and T. J. Sejnowski, "Independent component analysis using an extended infomax algorithm for mixed sub- and super-Gaussian sources," Neural Comput., vol. 11, pp. 606-633, 1999.

[25] C. T. Lin, W. C. Cheng, and S. F. Liang, "An on-line ICA-mixturemodel-based neural fuzzy network," IEEE Trans. Circuits Syst., Reg. Papers, vol. 52, no. 1, pp. 207-221, Jan. 2005.

[26] H. Ueno, M. Kaneda, and M. Tsukino, "Development of drowsiness detection system," in Proc. Veh. Navigation Inf. Syst. Conf., Aug. 1994, pp. $15-20$.

[27] T. P. Jung, C. Humphries, T. W. Lee, S. Makeig, M. J. McKeown, V. Iragui, and T. J. Sejnowski, "Extended ICA removes artifacts from electroencephalographic recordings," Adv. Neur. Inf. Process. Syst., vol. 10, pp. 894-900, 1998.

[28] T. P. Jung, S. Makeig, C. Humphries, T. W. Lee, M. J. McKeown, V. Iragui, and T. J. Sejnowski, "Removing electroencephalographic artifacts by blind source separation," Psychophysiol., vol. 37, pp. 163-78, 2000.

[29] T. P. Jung, S. Makeig, W. Westerfield, J. Townsend, E. Courchesne, and T. J. Sejnowski, "Analysis and visulization of single-trial event-related potentials," Human Brain Mapping, vol. 14, no. 3, pp. 166-85, 2001.

[30] A. Yamazaki, T. Tajima, and K. Matsuoka, "Convolutive independent component analysis of EEG data," in Proc. Annu. Conf. SICE, Aug. 2003, vol. 2, pp. 1227-1231.

[31] A. Meyer-Base, D. Auer, and A. Wismueller, "Topographic independent component analysis for fMRI signal detection," in Proc. Int. Joint Conf. Neural Netw., 2003, vol. 1, pp. 601-605.

[32] M. Naganawa, Y. Kimura, K. Ishii, K. Oda, K. Ishiwata, and A. Matani, "Extraction of a plasma time-activity curve from dynamic brain pet images based on independent component analysis," IEEE Trans. Biomed. Eng., vol. 52, no. 2, pp. 201-210, Feb. 2005.

[33] R. Liao, J. L. Krolik, and M. J. McKeown, "An information-theoretic criterion for intrasubject alignment of FMRI time series: Motion corrected independent component analysis," IEEE Trans. Med. Imaging, vol. 24, no. 1, pp. 29-44, Jan. 2005.

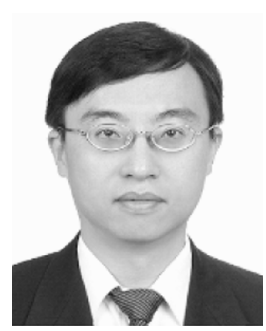

Chin-Teng Lin (F'05) received B.S. degree from the National Chiao-Tung University (NCTU), Hsinchu, Taiwan, R.O.C., and the Ph.D. degrees in electrical engineering from Purdue University, Lafayette, IN, in 1986 and 1992, respectively.

$\mathrm{He}$ is currently the Chair Professor of Electrical and Computer Engineering at NCTU. Dr. Lin has published over 90 journal papers.

Dr. Lin is an IEEE Fellow for his contributions to biologically inspired information systems.

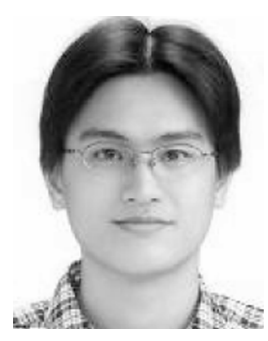

Li-Wei Ko received the B.S. degree in mathematics from National Chung Cheng University, Chiayi, Taiwan, R.O.C., and the M.S. degree in educational measurement and statistics from National Taichung University, Taichung, Taiwan, R.O.C., in 2001 and 2004, respectively. $\mathrm{He}$ is currently working toward the Ph.D. degree in electrical and control engineering at National Chiao-Tung University, Hsinchu, Taiwan, R.O.C.

His research interests are in the area of pattern recognition, machine learning, fuzzy neural networks, and biomedical signal processing.

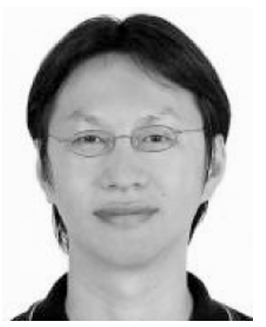

I-Fang Chung received the B.S. and M.S. degrees in control engineering, and the Ph.D. degree in electrical and control engineering from the National Chiao-Tung University (NCTU), Hsinchu, Taiwan, R.O.C., in 1993, 1995, and 2000, respectively.

Since 2004, he has served an Assistant Professor at the Institute of Bioinformatics, National Yang-Ming University, Taipei, Taiwan, R.O.C. His current research interests are bioinformatics, machine learning, and biomedical engineering.

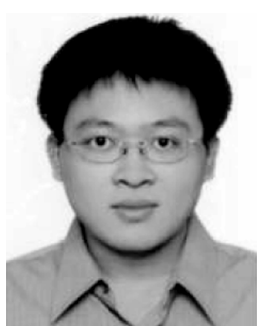

Teng-Yi Huang received the B.S. degree in electrical engineering from National Central University, Taiwan, R.O.C., and M.S. degree in electrical and control engineering from National Chiao-Tung University (NCTU), Hsinchu, Taiwan, R.O.C., in 2002, and 2004, respectively. He is currently working toward the Ph.D. degree at NCTU.

His research interests are in the areas of biomedical signal processing, biofeedback control, and virtual reality technology.

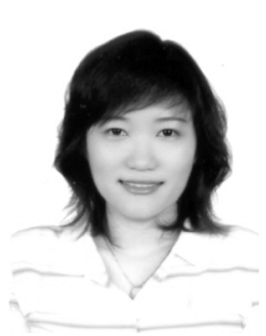

Yu-Chieh Chen received the B.S. degree in electrical engineering from Yuan-ze University, Taoyuan, Taiwan, R.O.C., and the M.S. degree in electrical engineering from Chang-Gung University, Taoyuan, Taiwan, R.O.C. in 2001 and 2003, respectively. She is currently working toward the Ph.D. degree at the National Chiao-Tung University, Hsinchu, Taiwan, R.O.C.

Her research interests are in the areas of artificial neural networks and biomedical signal processing.

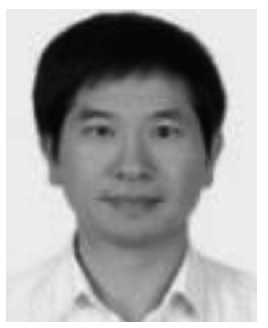

Tzyy-Ping Jung received the B.S. degree in electronics engineering from the National Chiao-Tung University, Hsinchu, Taiwan, R.O.C. in 1984, and the M.S. and Ph.D. degrees in electrical engineering from The Ohio State University, Columbus, in 1989 and 1993, respectively.

He was a Research Associate at the Computational Neurobiology Laboratory, The Salk Institute, San Diego, CA. He is currently an Associate Research Professor in the Institute for Neural Computation of the University of California, San Diego. He is also the Associate Director of the Swartz Center for Computational Neuroscience at UCSD. His research interests are in the areas of biomedical signal processing, cognitive neuroscience, artificial neural networks, time-frequency analysis of human electroencephalogram, functional neuroimaging, and neural human system interfaces.

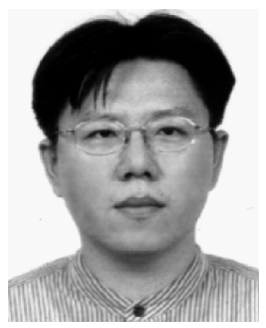

Sheng-Fu Liang received the B.S. and M.S. degrees in control engineering, and the Ph.D. degree in electrical and control engineering from the National Chiao-Tung University (NCTU), Hsinchu, Taiwan, R.O.C., in 1994, 1996, and 2000, respectively.

In 2005, he joined the Department of Biological Science and Technology, NCTU, where he served as an Assistant Professor. Currently, he is an Assistant Professor in the Department of Computer Science and Information Engineering, National Cheng-Kung University, Tainan, Taiwan, R.O.C. His current research interests are biomedical engineering and biomedical signal/image processing. 